\title{
Wide-angle electron beams from laser-wakefield accelerators
}

\author{
E. Brunetti ${ }^{1}$, X. Yang ${ }^{1, \dagger}$, F.Y. Li ${ }^{1}$, D. Reboredo Gil ${ }^{1}$, G.H. Welsh ${ }^{1}$, S. Cipiccia ${ }^{1}$, B. Ersfeld ${ }^{1}$, \\ D.W. Grant ${ }^{1}$, P.A. Grant ${ }^{1}$, M.R. Islam ${ }^{1}$, M. Shahzad ${ }^{1}$, M.P. Tooley ${ }^{1}$, G. Vieux ${ }^{1,2}$, \\ S.M. Wiggins ${ }^{1}$, Z.M. Sheng ${ }^{1,3}$, and D.A. Jaroszynski ${ }^{1}$ \\ ${ }^{1}$ SUPA, Department of Physics, University of Strathclyde, Glasgow, G4 0NG, UK \\ ${ }^{2}$ Institute of Physics of the ASCR, ELI-Beamlines, Na Slovance 2, 18221 Prague, Czech \\ Republic \\ ${ }^{3}$ Laboratory of Laser Plasmas and Department of Physics and Astronomy, Shanghai Jiao Tong \\ University, Shanghai 200240, China \\ ${ }^{\dagger}$ Present address: Department of Physics, Capital Normal University, Beijing 100048, China
}

\begin{abstract}
Advances in laser technology have driven the development of laser-wakefield accelerators, compact devices that are capable of accelerating electrons to $\mathrm{GeV}$ energies over centimetre distances by exploiting the strong electric field gradients arising from the interaction of intense laser pulses with an underdense plasma. A side-effect of this acceleration mechanism is the production of high-charge, low-energy electron beams at wide angles. Here we present an experimental and numerical study of the properties of these wide-angle electron beams, and show that they carry off a significant fraction of the energy transferred from the laser to the plasma. These high-charge, wide-angle beams can also cause damage to laser-wakefield accelerators based on capillaries, as well as become source of unwanted bremsstrahlung radiation.
\end{abstract}

Keywords: Laser-wakefield acceleration, low-energy high-charge electron beams, laser-driven particle accelerators, SPIE Proceedings

\section{INTRODUCTION}

Laser-wakefield acceleration is a promising technique to produce high-energy electron beams by high-power lasers available now in many university-scale laboratories. ${ }^{1-4}$ The radiation pressure of an intense laser pulse propagating in an underdense plasma or ionized gas expels background electrons radially outward, while leaving the heavier ions stationary. This results in the formation of an ion cavity ("bubble") trailing behind the laser pulse, with the electrons forming a dense sheath around the bubble. This charge separation creates a strong electric field that pulls electrons back after the laser passes, producing a large amplitude travelling plasma wave. Background electrons that enter the bubble and become trapped inside it are exposed to accelerating fields on the order of $100 \mathrm{GV} / \mathrm{m}$, which is about three orders of magnitude greater than what is achievable in conventional radio-frequency cavities. ${ }^{5}$ This results in the production of $\mathrm{MeV}-\mathrm{GeV}$ scale electron beams over short distances. For example, electron beams of energy in excess of $4 \mathrm{GeV}$ have been obtained in a $9 \mathrm{~cm}$ plasma waveguide powered by a $300 \mathrm{TW}$ laser pulse. ${ }^{6}$

Only a small fraction of plasma electrons can be injected into the bubble and laser-wakefield accelerators typically produce beams with a small charge at $\mathrm{pC}$ level, which is desirable when high-quality beams with short bunch length, narrow energy spread and low emittance are required, for novel radiation sources. ${ }^{7-14}$ There are, however, applications such as non-destructive-testing, ultra-fast studies in condensed matter, radiolysis, isotope production and radiotherapy that require high-charge, low-energy electron beams. ${ }^{15-19}$ Ionization-assisted injection in high- $\mathrm{Z}$ or clustering gas targets has been explored to boost the charge to $100 \mathrm{~s} \mathrm{pC}$ at $10 \mathrm{~s}-100 \mathrm{~s} \mathrm{MeV}$ energies. ${ }^{20-22}$ At even lower energies, high-charge beams can be obtained by exploiting the little known property of laser-wakefield accelerators that intrinsically leads to $\mathrm{MeV}$ electrons at wide angles as part of the bubble formation process. ${ }^{23-25}$ These oblique electrons typically have 1-2 MeV mean energy and nC-level total charge,

E-mail: enrico.brunetti@strath.ac.uk, d.a.jaroszynski@strath.ac.uk 
and are distributed in a broad cone of $30^{\circ}-50^{\circ}$ half-angle with respect to the laser-propagation axis. Although potentially useful for some applications, these wide-angle beams can also have undesirable effects. If not properly dumped, they can produce a high flux of unwanted bremsstrahlung radiation and noise. They can damage equipments placed close to the accelerator, which is a particular concern for a set-up based on capillaries. These high-charge electron beams can also carry a large amount of energy out of the plasma, limiting the overall efficiency of the accelerator.

Here we present an overview of the properties of the wide-angle electron beams. In Section 2 we report numerical simulations of the mechanism leading to the emission of oblique electrons, and highlight differences in the spectral and angular distributions of wide-angle beams depending on whether high-energy forward electron beams are also produced. In Section 3 we present experimental measurements of the properties of wide-angle electron beams. In Section 4 we briefly discuss the results obtained.

\section{NUMERICAL SIMULATIONS}

The interaction of a high-power laser beam with a helium gas jet is simulated both in 3D and 2D geometry using the particle-in-cell (PIC) code OSIRIS. ${ }^{26}$ The laser pulse propagates along the $x_{1}$ direction and has a longitudinal sine-squared shape with full width at half maximum that is varied between 20 and $30 \mathrm{fs}$. The transverse profile is Gaussian and the beam size $w_{0}$ (radius at the $1 / e^{2}$ intensity point) is varied between 5 and $10 \mu \mathrm{m}$. The laser intensity $I_{0}$ is expressed in terms of the normalized vector potential $a_{0}=8.5 \times 10^{-10} \lambda_{L}[\mathrm{\mu m}]\left(I_{0}\left[\mathrm{~W} / \mathrm{cm}^{2}\right]\right)^{1 / 2}$, with $\lambda_{L}$ being the laser wavelength. Here, $\lambda_{L}=0.8 \mu \mathrm{m}$. The focal plane is located at the entrance of a preionized plasma with isotropic thermal temperature of $20 \mathrm{eV}$ and with a constant density profile preceded by a linear up-ramp of length $40 \mu \mathrm{m}$ in $3 \mathrm{D}$ and $60 \mu \mathrm{m}$ in $2 \mathrm{D}$. The laser is linearly polarized in the $x_{2}$ (horizontal) direction for 3D simulations and in the $x_{3}$ direction for 2D simulations. Most 3D simulations are performed in a $50 \mu \mathrm{m} \times 40 \mu \mathrm{m} \times 40 \mu \mathrm{m}$ box with $1560 \times 160 \times 160$ cells. Simulations are also performed in $2 \mathrm{D}$ geometry for several configurations to ensure that the properties of oblique electrons are consistent against varying grid size and time resolution. Most 2D results are obtained for a $60 \mu \mathrm{m} \times 80 \mu \mathrm{m}$ moving window with $3450 \times 2400$ cells. In the calculation of the properties of oblique electrons, high-energy forward electrons and low-energy plasma electrons are filtered out by selecting only those electrons with longitudinal momentum $1.4<p_{1} / m c<50$ and transverse position $r>5-8 \mu \mathrm{m}$, depending on the bubble size.

The dynamics of electrons ejected from the plasma at wide angles is illustrated in Figure 1, obtained from 3D PIC simulations of a $20 \mathrm{fs}$ laser pulse with $a_{0}=2$ and spot size $w_{0}=5 \mu \mathrm{m}$ interacting with a plasma of density $2 \times 10^{19} \mathrm{~cm}^{-3}$. Sample trajectories are provided in Figure 1a, superimposed on a snapshot of the electron density after $260 \mu \mathrm{m}$ propagation distance, when a train of five non-linear wakes are visible behind the laser pulse. Electrons can reach energies up to about $3.5 \mathrm{MeV}$, as shown in Figure 1b. The acceleration mechanism can be inferred from the evolution of electron position and momentum, detailed in figures 1c-1h. The horizontal components $x_{2}$ and $p_{2}$, which are parallel to the laser polarization direction, indicate that electrons initially oscillate in the laser electric field with little energy gain. Efficient acceleration is instead driven by the strong electrostatic forces experienced as the electrons stream in the dense sheath surrounding the bubble. Because of the cylindrical symmetry, horizontal and vertical components of the momentum obtain similar values at the end of the interaction.

The magnitude of the accelerating electric field is about $10-100 \mathrm{GV} / \mathrm{m}$ in all directions, with typical acceleration lengths of $10-20 \mu \mathrm{m}$ in the laser propagation direction $x_{1}$ and 5-10 $\mathrm{mm}$ in the transverse directions $x_{2}$ and $x_{3}$. This indicates that oblique electrons can travel along with the bubble and gain a larger longitudinal momentum, whereas the transverse acceleration is limited by the bubble size. Figure 2a shows that the highest energies are gained for electrons initially located off-axis by an amount close to the bubble radius, which is about $4 \mu \mathrm{m}$ for the parameters of this simulation. Figure $2 \mathrm{~b}$ shows that there is a correlation between the final energy and ejection angle, with higher energy electrons emitted closer to the laser propagation axis.

The properties of the accelerating structure can be tuned by varying laser and plasma parameters. ${ }^{27}$ The strength of the electric field inside and around the bubble typically increases with the plasma density, but with a corresponding decrease in bubble size, and therefore in acceleration length for oblique electrons. Higher electron energies are more likely to be obtained for low plasma densities and large laser $a_{0}$, where the bubble is bigger. 

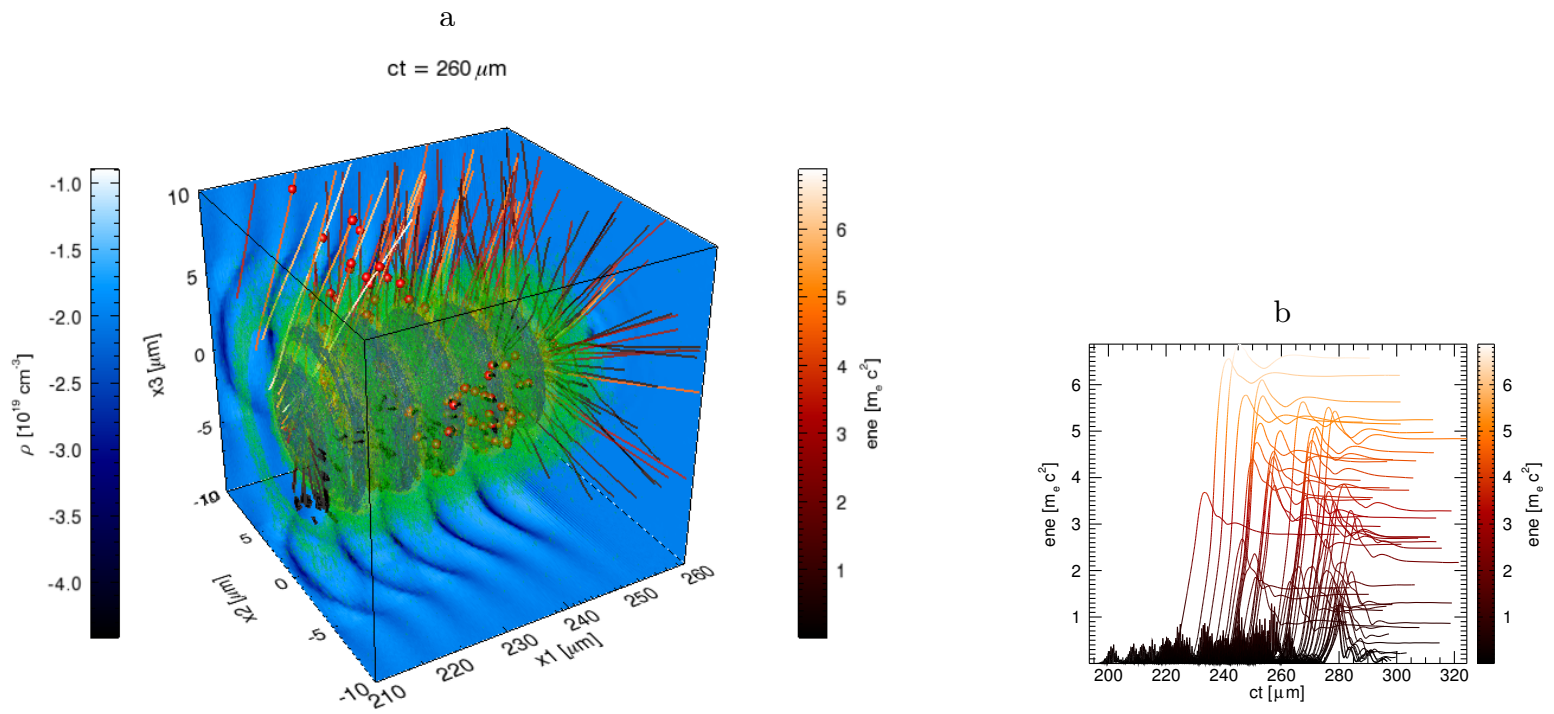

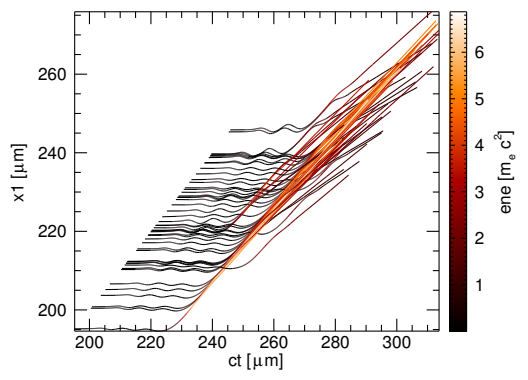

$\mathrm{f}$

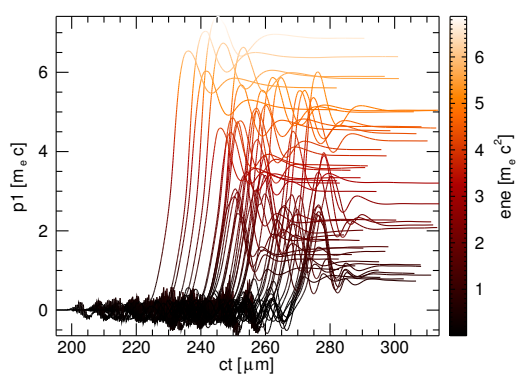

d

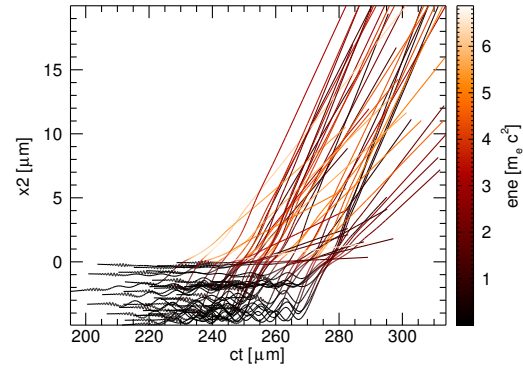

$\mathrm{g}$

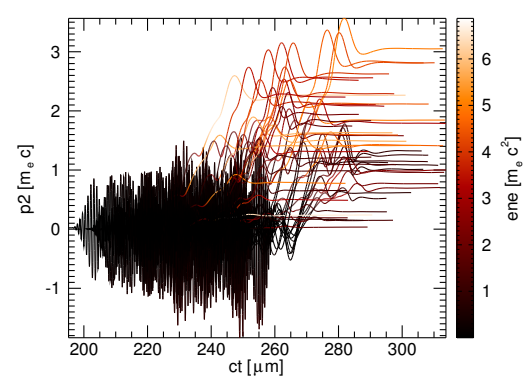

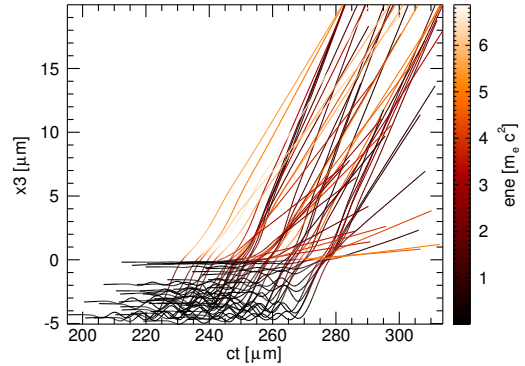

$\mathrm{h}$

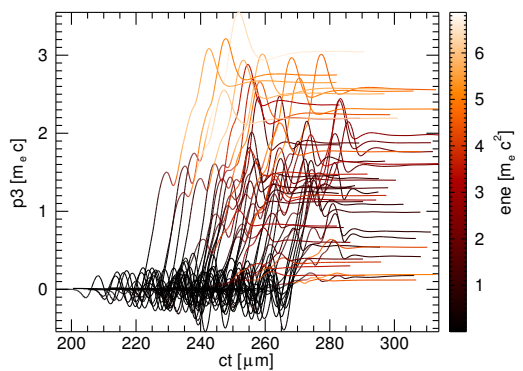

Figure 1: Properties of sample electrons ejected at wide angles obtained from 3D PIC simulation. a) Trajectories of 200 electrons superimposed on a snapshot of the electron density after $260 \mu \mathrm{m}$ propagation. Plots of the time evolution of 50 sample electrons illustrating b) electron energy, c) longitudinal coordinate $x_{1}$, d) horizontal coordinate $x_{2}$, e) vertical coordinate $x_{3}$, f) longitudinal momentum $p_{1} / m_{e} c$, g) horizontal momentum $p_{2} / m_{e} c$, h) vertical momentum $p_{3} / m_{e} c$. The line color represents the electron energy (in units of $m_{e} c^{2}=0.511 \mathrm{MeV}$ ). 


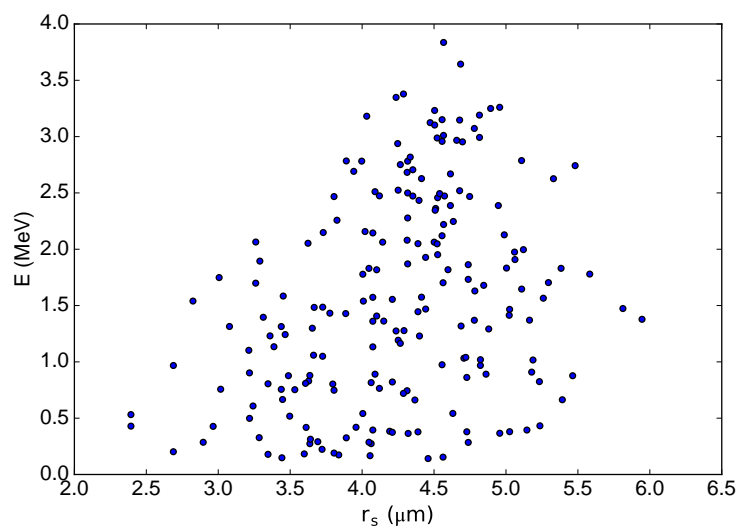

$\mathrm{b}$

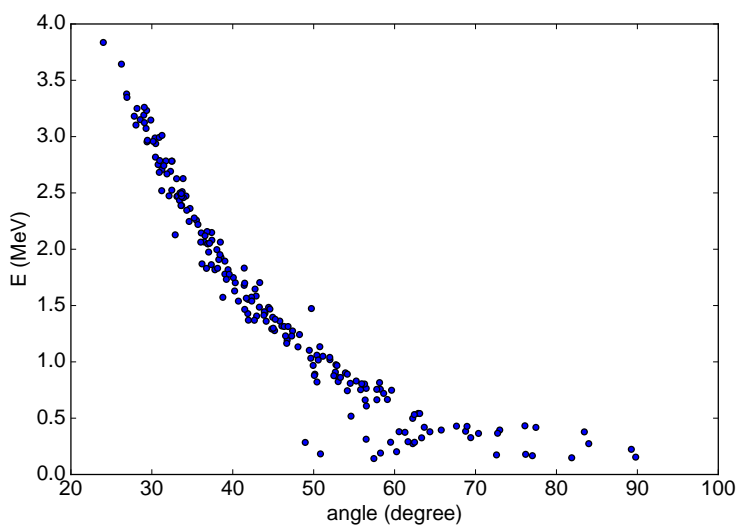

Figure 2: Final energy of 200 sample electrons ejected at wide angles obtained from the same 3D PIC simulation of Figure 1. a) Final energy versus initial radial displacement from the laser propagation axis. b) Final energy versus ejection angle.

This energy boost mostly affects the longitudinal momentum component, because of longer acceleration lengths, and the most energetic electrons are emitted at small angles, close to the laser propagation axis. On average, the properties of wide-angle electron beams depend weakly on laser and plasma parameters, especially at large angles, as shown below.

\subsection{Wide-angle electron beams and no injection}

In this section we describe the properties of wide-angle electron beams obtained from 3D PIC simulations for laser and plasma parameters that do not lead to the injection of background electrons inside the bubble. Therefore, high-energy forward electron beams are not produced. Examples of the angular and spectral distribution of wide-angle electron beams are presented in Figure 3 for three sets of plasma densities and laser parameters. Under these conditions injection does not occur and the accelerating structure evolves little during propagation. Oblique electrons are emitted in a hollow cone with angle of $20^{\circ}-50^{\circ}$ relative to the laser propagation axis, with a mean energy between 1 and $4 \mathrm{MeV}$. The electron energy depends on the angle, with the most energetic electrons emitted in the inner region of the cone, close to the laser propagation axis.

Plots in the left column are obtained for plasma density $n_{e}=5 \times 10^{18} \mathrm{~cm}^{-3}$, laser amplitude $a_{0}=3$, spot size $w_{0}=8 \mu \mathrm{m}$ and propagation distance $0.7 \mathrm{~mm}$. Electrons are ejected in a hollow cone with a mean angle of $32^{\circ}$ and $10^{\circ} \mathrm{rms}$ divergence. The mean energy is $3.9 \mathrm{MeV}$ with $90 \% \mathrm{rms}$ energy spread. The total charge is $2.7 \mathrm{nC}$. As expected for low densities and large $a_{0}$, the bubble is relatively big, in this case with a radius of approximately $8 \mathrm{\mu m}$ and speed of about $0.993 \mathrm{c}$. The bigger size allows for longer acceleration distances, with a boost in longitudinal momentum. As shown by the dashed curve in Figure 3d, the electron energy increases up to about $10 \mathrm{MeV}$ at small angles, close to the laser propagation axis. For angles larger than about $30^{\circ}$, the electron energy is less than $2 \mathrm{MeV}$.

Plots in the middle column correspond to plasma density $n_{e}=1 \times 10^{19} \mathrm{~cm}^{-3}$, laser amplitude $a_{0}=2$, spot size $w_{0}=7 \mu \mathrm{m}$ and propagation distance $0.7 \mathrm{~mm}$. The hollow area is wider, with no emission of more energetic electrons at angles smaller than $20^{\circ}$. The mean angle of the cone is $38^{\circ}$, with $6^{\circ} \mathrm{rms}$ divergence, $1.2 \mathrm{nC}$ total charge, $1.7 \mathrm{MeV}$ mean energy and $40 \% \mathrm{rms}$ energy spread. The bubble is smaller, about $5.5 \mu \mathrm{m}$ in radius, and the speed is again $0.993 \mathrm{c}$. The total charge is lower, because the plasma wave starts to decay after about $0.5 \mathrm{~mm}$ propagation and oblique electrons are no longer emitted (see Figure 4a).

Plots in the right column are obtained for plasma density $n_{e}=2 \times 10^{19} \mathrm{~cm}^{-3}$, laser $a_{0}=2$, spot size $w_{0}=5 \mu \mathrm{m}$ and propagation length $0.7 \mathrm{~mm}$. The mean angle of the cone is $36^{\circ}$, with $7^{\circ} \mathrm{rms}$ divergence, $3.3 \mathrm{nC}$ total charge, $1.7 \mathrm{MeV}$ mean energy and $50 \% \mathrm{rms}$ energy spread. The bubble radius is approximately $4 \mu \mathrm{m}$ and the speed is $0.992 c$. The electron beam properties are very similar to the previous case. 
a

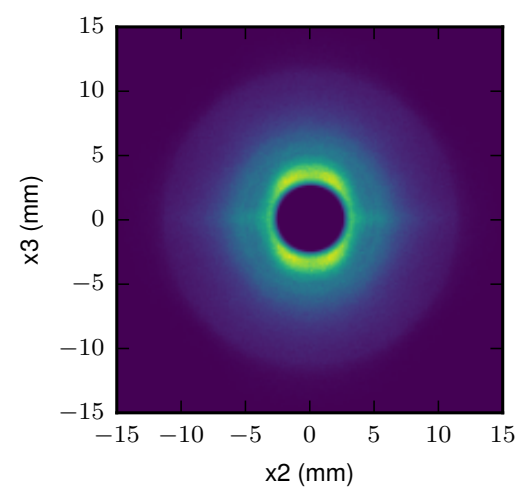

d

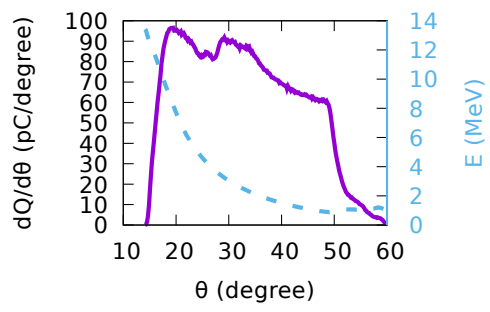

g

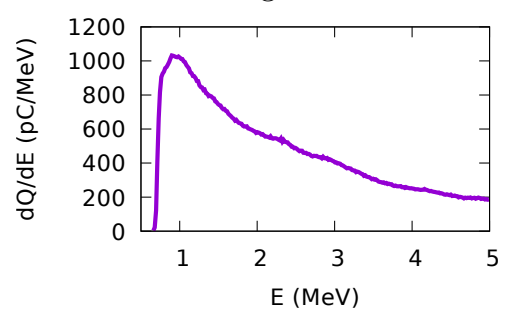

b

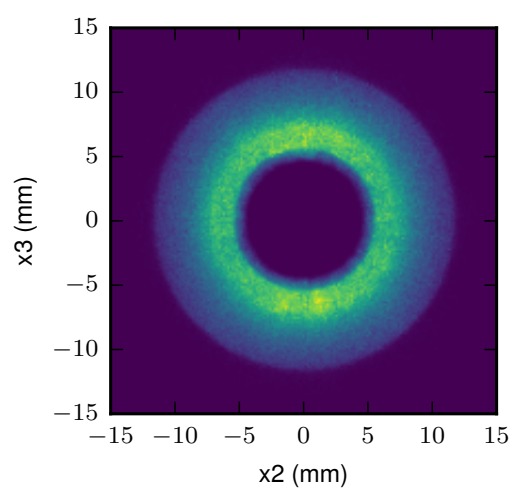

e

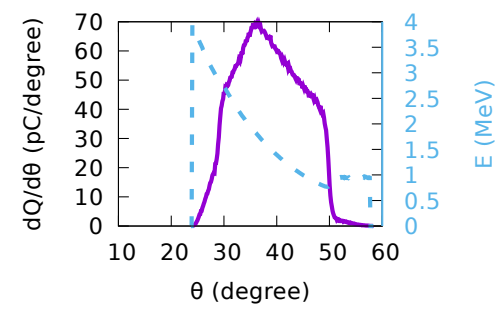

$\mathrm{h}$

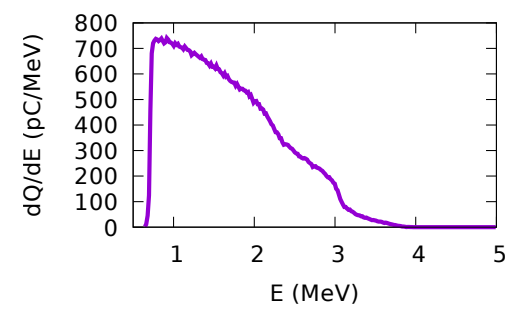

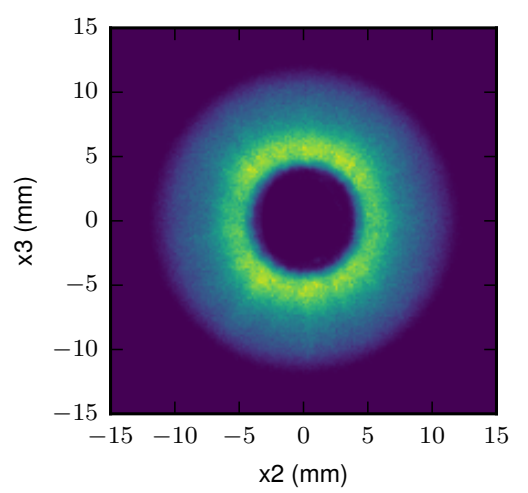

f

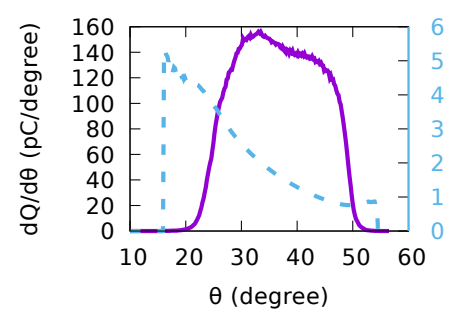

i

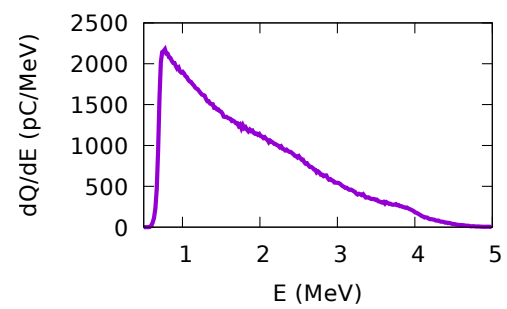

Figure 3: 3D PIC simulations of spatial (a-c), angular (d-f) and spectral (g-i) distribution of wide-angle electrons on a screen $10 \mathrm{~mm}$ downstream from the accelerator. The plasma density, laser intensity and spot size are $n_{e}=5 \times 10^{18} \mathrm{~cm}^{-3}, a_{0}=3, w_{0}=8 \mu \mathrm{m}$ (left column), $n_{e}=1 \times 10^{19} \mathrm{~cm}^{-3}, a_{0}=2, w_{0}=7 \mu \mathrm{m}$ (middle column) and $n_{e}=2 \times 10^{19} \mathrm{~cm}^{-3}, a_{0}=2, w_{0}=5 \mu \mathrm{m}$ (right column). Dashed curves in Figures (d-f) show the energy dependence on angle. 


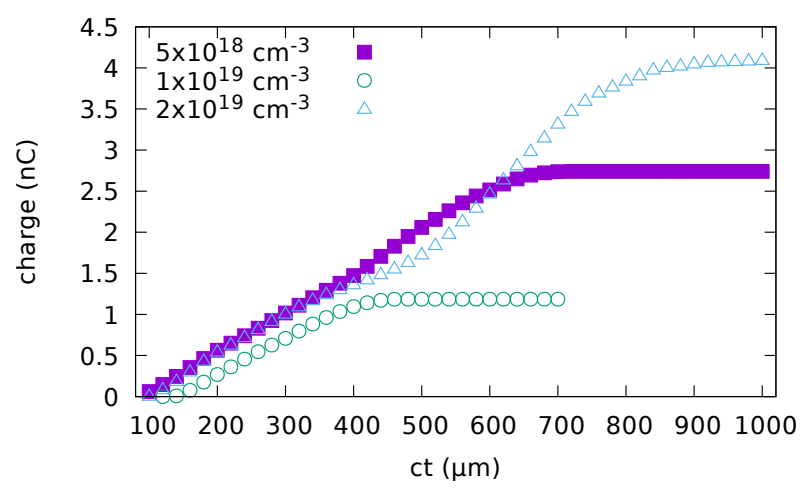

$\mathrm{b}$

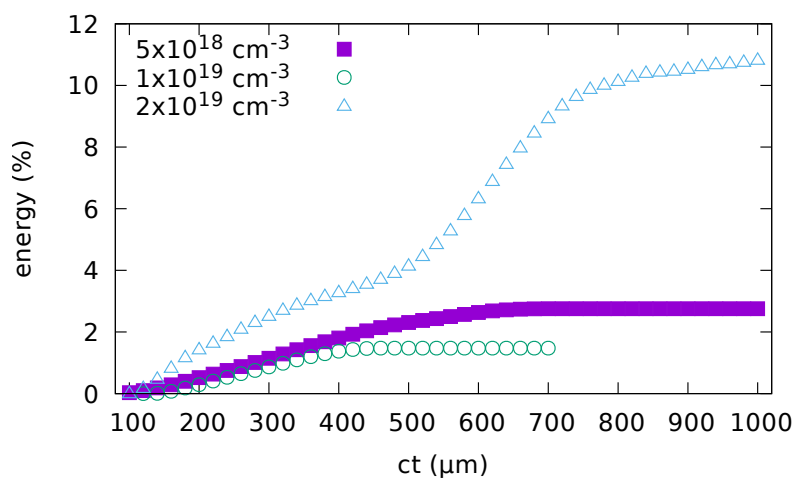

Figure 4: Emission rate of wide-angle electron beams obtained from 3D PIC simulations. Charge (a) and energy (b) of wide-angle electron beams versus propagation distance ct for $n_{e}=5 \times 10^{18} \mathrm{~cm}^{-3}$, laser $a_{0}=3$, spot size $w_{0}=8 \mu \mathrm{m}$ (squares), $n_{e}=1 \times 10^{19} \mathrm{~cm}^{-3}$, laser $a_{0}=2$, spot size $w_{0}=7 \mu \mathrm{m}(\operatorname{circles})$ and $n_{e}=2 \times 10^{19} \mathrm{~cm}^{-3}$, laser $a_{0}=2$, spot size $w_{0}=5 \mu \mathrm{m}$ (triangles).

The emission rate, plotted in Figure 4a, shows that the charge grows approximately linearly with propagation distance, until the plasma wave dies out. Because of the high charge, wide-angle electron beams can carry a significant fraction of the laser energy out of the plasma, as shown in Figure 4b, where the energy is expressed as a percentage of the initial laser energy, which is $391 \mathrm{~mJ}$ for $a_{0}=3, w_{0}=8 \mu \mathrm{m}, 134 \mathrm{~mJ}$ for $a_{0}=2, w_{0}=7 \mu \mathrm{m}$ and $68 \mathrm{~mJ}$ for $a_{0}=2, w_{0}=5 \mu \mathrm{m}$.

\subsection{Wide-angle electron beams and injection}

In this section we describe the properties of wide-angle electron beams obtained from 3D PIC simulations for laser and plasma parameters that lead to the injection of background electrons inside the bubble, with the production of high-energy forward electron beams. Examples of the angular and spectral distribution of wide-angle electron beams are presented in Figure 5 for three sets of plasma densities and laser parameters. Under these conditions the shape and size of the accelerating structure can change significantly during propagation and the hollow cone described in the previous section fills in with electrons with energy up to about $10 \mathrm{MeV}$.

Plots in the left column are obtained for a plasma density $n_{e}=1 \times 10^{19} \mathrm{~cm}^{-3}$, laser amplitude $a_{0}=4$, spot size $w_{0}=7 \mu \mathrm{m}$ and propagation length $1 \mathrm{~mm}$. The mean angle of the cone is $40^{\circ}$, with $10^{\circ} \mathrm{rms}$ divergence, $12.8 \mathrm{nC}$ total charge, $2 \mathrm{MeV}$ mean energy and $110 \%$ rms energy spread. The average bubble size over the propagation distance is approximately $8 \mu \mathrm{m}$ and the speed is $0.991 \mathrm{c}$. In comparison with the results obtained in the previous section for no injection, the charge is higher, especially at large angles, where electrons have lower energy. This is reflected in the mean energy, which is lower, since high-energy electrons ejected close to the laser propagation axis represent only a small fraction of the total charge.

Plots in the middle column are obtained for plasma density $n_{e}=2 \times 10^{19} \mathrm{~cm}^{-3}$, laser amplitude $a_{0}=3$, spot size $w_{0}=7 \mu \mathrm{m}$ and propagation length $0.5 \mathrm{~mm}$. The mean angle of the cone is $38^{\circ}$, with $13^{\circ} \mathrm{rms}$ divergence, $10 \mathrm{nC}$ total charge, $1.8 \mathrm{MeV}$ mean energy and $115 \% \mathrm{rms}$ energy spread. The average bubble size over the propagation distance is approximately $6 \mu \mathrm{m}$ and the bubble travels at a speed of $0.976 \mathrm{c}$. For these parameters the cone completely fills in with electrons. A horizontal band with higher charge density is visible parallel to the laser

polarization direction. A similar feature is observed in the charge distribution measured experimentally and presented in Section 3.

Plots in the right column are obtained for plasma density $n_{e}=4 \times 10^{19} \mathrm{~cm}^{-3}$, laser amplitude $a_{0}=3$, spot size $w_{0}=7 \mu \mathrm{m}$ and propagation length $0.5 \mathrm{~mm}$. The mean angle of the cone is $32^{\circ}$, with $14^{\circ} \mathrm{rms}$ divergence, $17 \mathrm{nC}$ total charge, $2 \mathrm{MeV}$ mean energy and $115 \%$ rms energy spread. The mean bubble size over the propagation distance is approximately $5 \mu \mathrm{m}$ and the bubble travels at a speed of $0.952 c$.

The emission rate versus propagation distance is plotted in Figure 6a, which exhibits a wider variety of trends in comparison with the results obtained for no injection (Figure 4a). High charges can be produced over 
a

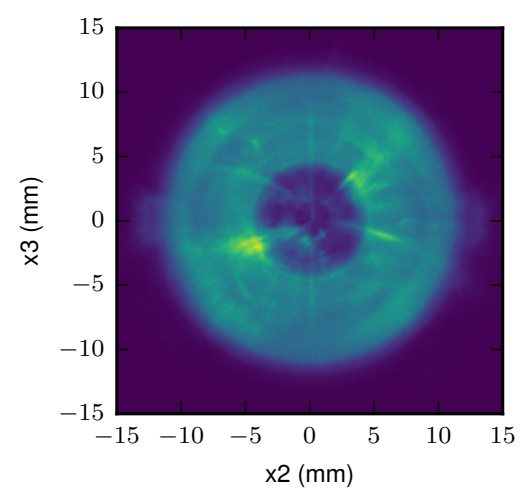

d

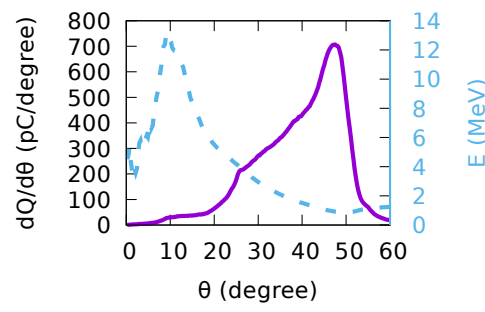

g

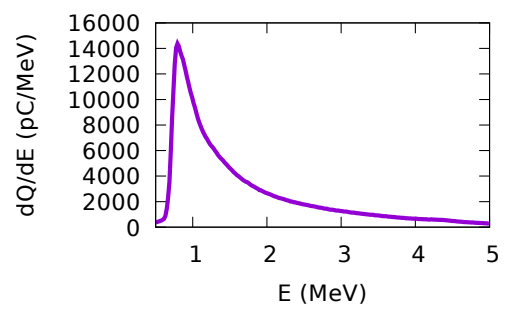

b

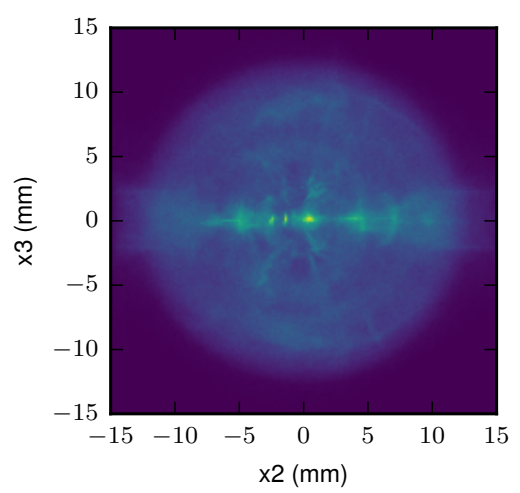

e

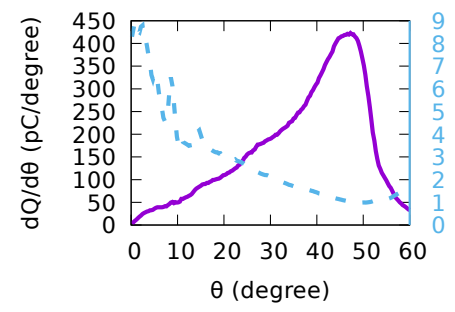

$\mathrm{h}$

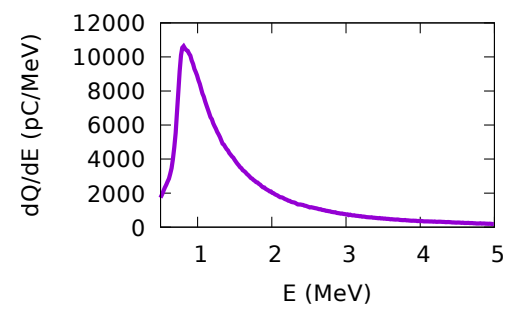

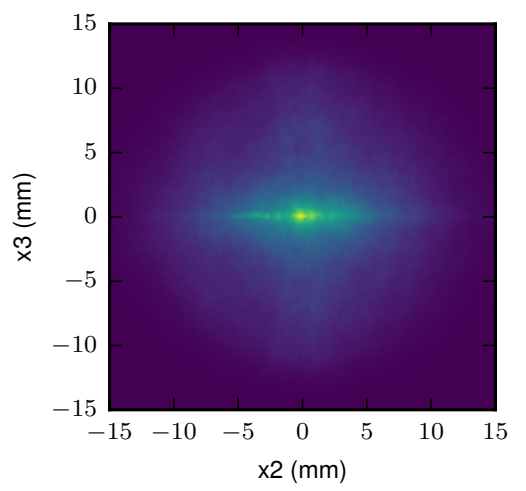

f

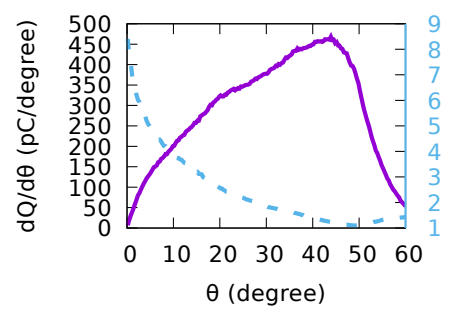

i

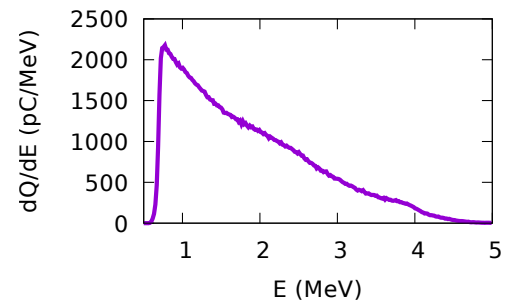

Figure 5: 3D PIC simulations of spatial (a-c), angular (d-f) and spectral (g-i) distribution of wide-angle electrons on a screen $10 \mathrm{~mm}$ downstream from the accelerator. The plasma density, laser intensity and spot size are $n_{e}=1 \times 10^{19} \mathrm{~cm}^{-3}, a_{0}=4, w_{0}=7 \mu \mathrm{m}$ (left column), $n_{e}=2 \times 10^{19} \mathrm{~cm}^{-3}, a_{0}=3, w_{0}=7 \mu \mathrm{m}$ (middle column) and $n_{e}=4 \times 10^{19} \mathrm{~cm}^{-3}, a_{0}=3, w_{0}=7 \mu \mathrm{m}$ (right column). Dashed curves in Figures (d-f) show the energy dependence on angle. 
a

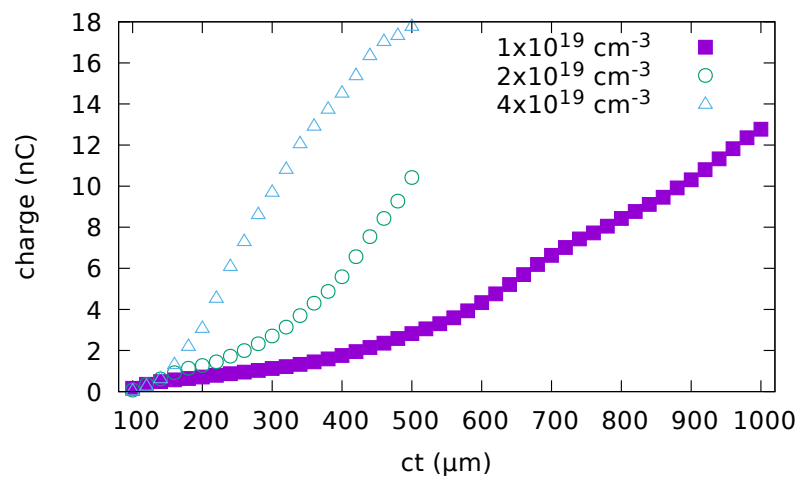

$\mathrm{b}$

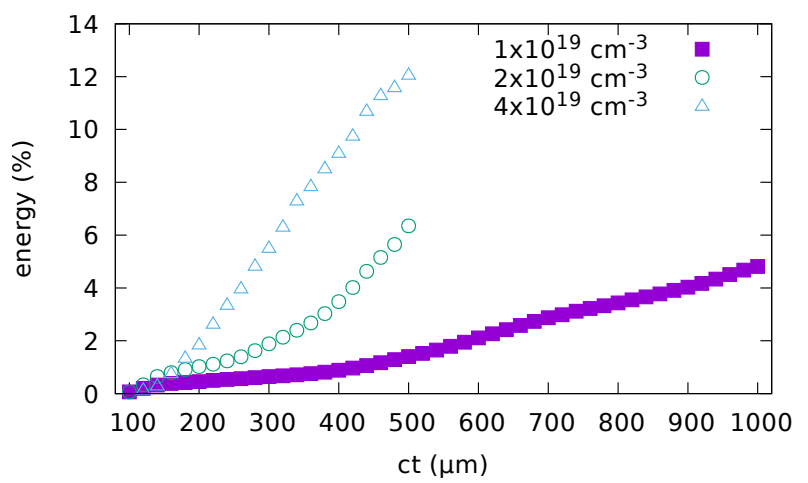

Figure 6: Emission rate of wide-angle electron beams obtained from 3D PIC simulations. a) Charge and b) energy versus propagation distance $c t$ for $n_{e}=1 \times 10^{19} \mathrm{~cm}^{-3}$, laser $a_{0}=4$, spot size $w_{0}=7 \mu \mathrm{m}$ (squares), $n_{e}=2 \times 10^{19} \mathrm{~cm}^{-3}$, laser $a_{0}=3$, spot size $w_{0}=7 \mu \mathrm{m}$ (circles) and $n_{e}=4 \times 10^{19} \mathrm{~cm}^{-3}$, laser $a_{0}=3$, spot size $w_{0}=7 \mu \mathrm{m}$ (triangles).

short distances at high densities. At low densities the growth rate is slower, but it can be maintained for longer distances. Typically, the total charge of wide-angle electron beams grows linearly with density and quadratically with laser amplitude $a_{0}$, or linearly with intensity, assuming that the frequency does not change much. ${ }^{25}$

Wide-angle electron beams can carry a significant fraction of the laser energy out of the plasma, as shown in Figure $6 \mathrm{~b}$, where the energy is expressed as a percentage of the initial laser energy, which is $535 \mathrm{~mJ}$ for $a_{0}=4$, $w_{0}=7 \mu \mathrm{m}$ and $301 \mathrm{~mJ}$ for $a_{0}=3, w_{0}=7 \mu \mathrm{m}$.

\section{EXPERIMENTAL RESULTS}

The properties of wide-angle electron beams have been experimentally measured at the Advanced Laser-Plasma High-energy Accelerators towards X-rays (ALPHA-X) beam line. ${ }^{28}$ A Ti:sapphire Chirp Pulse Amplification (CPA) laser system delivers $35 \mathrm{fs}, 800 \mathrm{~nm}$ pulses with on-target energy of $900 \mathrm{~mJ}$. The laser beam, polarized in the horizontal plane $(x)$, is focused onto a gas jet by an $f / 18$ spherical mirror to a vacuum spot size of $20 \mu \mathrm{m}$ (radius at the $1 / e^{2}$ intensity point). The supersonic He gas jet is produced by a $2 \mathrm{~mm}$ diameter nozzle with plasma density $1-2 \times 10^{19} \mathrm{~cm}^{-3}$. The laser peak intensity is $2 \times 10^{18} \mathrm{~W} / \mathrm{cm}^{2}$, corresponding to $a_{0} \approx 1$, which grows to $a_{0}>3$ after about $0.5 \mathrm{~mm}$ propagation due to self-focusing. This results in the formation of a plasma channel with radius approximately $5 \mu \mathrm{m}$.

The spatial distribution of wide-angle electron beams is measured on a $21 \times 17.5 \mathrm{~cm}^{2}$ EBT2 Gafchromic dosimetry film ${ }^{29}$ placed $4 \mathrm{~cm}$ in front of the nozzle. A $2 \mathrm{~cm}$ diameter hole is cut at the center to let the laser pass through. The distribution recorded after accumulating 70 shots is presented in Figure $7 \mathrm{a}$, where a ring is visible at a $40^{\circ}$ angle from the laser propagation axis, with a width of $12^{\circ}$. A band containing a higher charge density is visible along the horizontal axis, which is parallel to the laser polarization direction. The clipping at the bottom and left is caused by the gas nozzle mount. The total charge in the ring is estimated to be $10 \mathrm{nC}$ per shot from Monte-Carlo simulations with the code FLUKA. ${ }^{30,31}$ Figure $7 \mathrm{~b}$ shows the mean horizontal $\left(\theta_{x}\right)$ and vertical $\left(\theta_{y}\right)$ angle for the middle-right section of the cone, which is measured on a LANEX screen for 200 consecutive shots. The average ejection angle is $(41 \pm 1)^{\circ}$, with $\mathrm{rms}$ horizontal divergence of $(11.0 \pm 0.5)^{\circ}$ and $20 \%$ variation in charge.

The energy of wide-angle electron beams is measured with a spectrometer composed of two dipole magnets separated by a $1 \mathrm{~cm}$ gap and producing a $200 \mathrm{mT}$ magnetic field, for an operating range of approximately $0.5^{-}$ $3 \mathrm{MeV}$. A $2 \mathrm{~mm}$ wide copper slit is placed in front of the magnets and a LANEX screen wrapped in thin Al foil is on the back. The spectrometer is placed $5 \mathrm{~cm}$ from the nozzle and oriented at an angle of $45^{\circ}$ to the laser axis. A sample single-shot spectrum is presented in Figure 8a, corresponding to a mean energy of $1.1 \mathrm{MeV}$ with $55 \%$ energy spread. The tilt indicates that the energy is higher in the inner section of the cone, close to the laser polarization axis $\left(\theta_{y}=0\right)$, in agreement with the theoretical predictions reported in Section 2. The energy and 
a

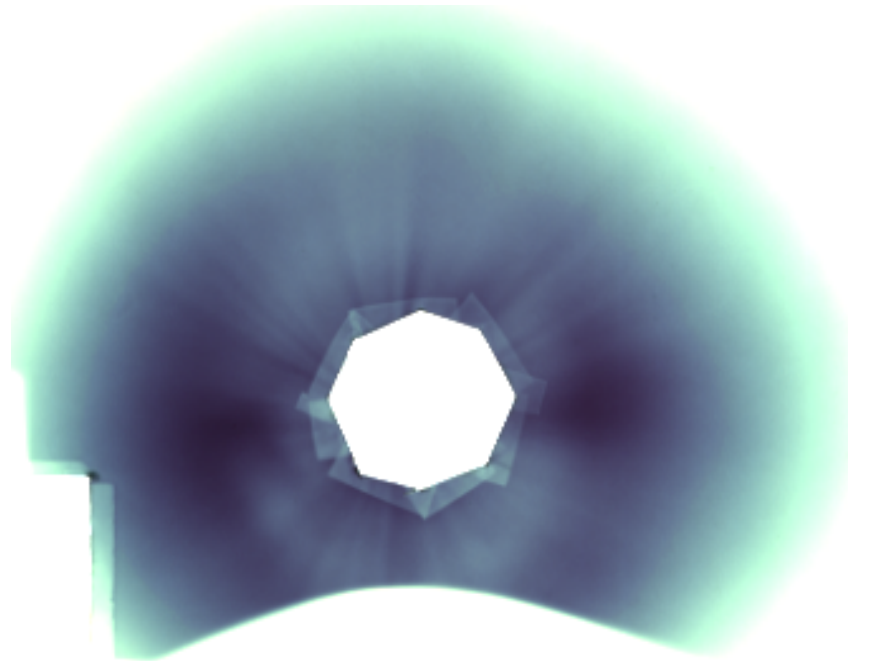

$\mathrm{b}$

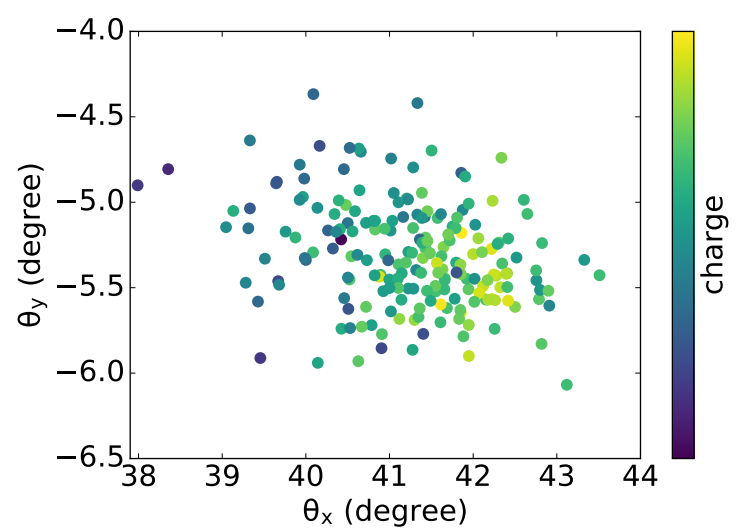

Figure 7: Experimental characterization of wide-angle electron beams. a) Spatial distribution measured by accumulating 70 shots on a Gafchromic film placed $4 \mathrm{~cm}$ after the nozzle. b) Mean horizontal and vertical angle for 200 consecutive shots measured on a LANEX screen.

a

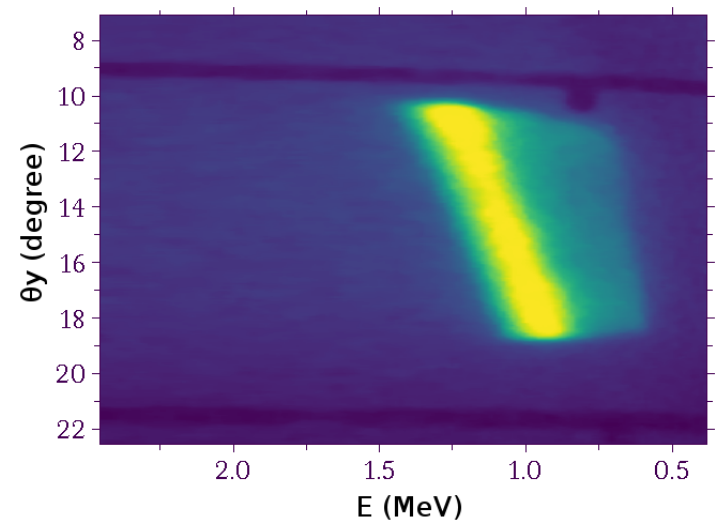

b

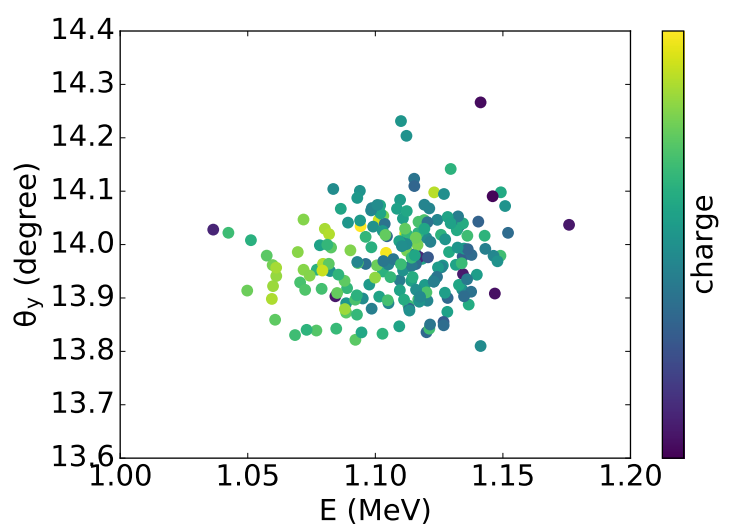

Figure 8: Energy measurements of wide-angle electron beams obtained with a magnetic spectrometer. a) Single shot spectrum. b) Mean energy and vertical angle for 200 consecutive shots. 
vertical angle for 200 consecutive shots are shown in Figure $8 \mathrm{~b}$. An analysis of 984 shots indicates that the mean energy is $(1.10 \pm 0.25) \mathrm{MeV}$, with an energy spread of $(0.52 \pm 0.03) \mathrm{MeV}$ and $34 \%$ variation in charge.

\section{DISCUSSION}

Laser-wakefield accelerators have the intrinsic property of emitting a low-energy nC-level electron beam in a wide cone aligned with the laser propagation direction. This cone is hollow when injection into the bubble does not occur and high-energy forward electron beams are not produced. When injection occurs, the cone fills in with more energetic electrons. In both cases, a correlation is observed between ejection angle and electron energy. For angles greater than approximately $30^{\circ}$, the electron energy is about $1-2 \mathrm{MeV}$. When moving towards the central axis of the cone, the energy increases. For example, it is about $4 \mathrm{MeV}$ at $20^{\circ}$ and can be greater than $10 \mathrm{MeV}$ for angles smaller than $10^{\circ}$. Changes in laser and plasma parameters mostly affect the inner part of the cone, which can be empty or filled with the most energetic electrons, whereas the outer section is stable, with little variation in energy at a given angle. The charge, however, can vary substantially across the entire cone, typically increasing with plasma density and laser amplitude $a_{0}$. Hot spots corresponding to higher charge density can also appear, with patterns depending on laser and plasma parameters.

Wide-angle electron beams can be useful for applications requiring a high flux of electrons or bremsstrahlung photons over a large area, but can also be source of unwanted noise or radiation. The bremsstrahlung photon spectrum produced by a $2 \mathrm{MeV}$ electron beam with $50 \%$ energy spread after interaction with different materials of the same thickness $(5 \mathrm{~mm})$ is shown in Figure 9. The integrated flux over all energies generated, for example, in aluminium, stainless steel and copper is about $5 \times 10^{-2}$ photons/primary, which corresponds to more than $3 \times 10^{7}$ photons per shot for a charge of $100 \mathrm{pC}$. Since wide-angle beams are produced with high-repeatability at each laser shot, the amount of radiation emitted is substantial both in a single shot and accumulated over time.

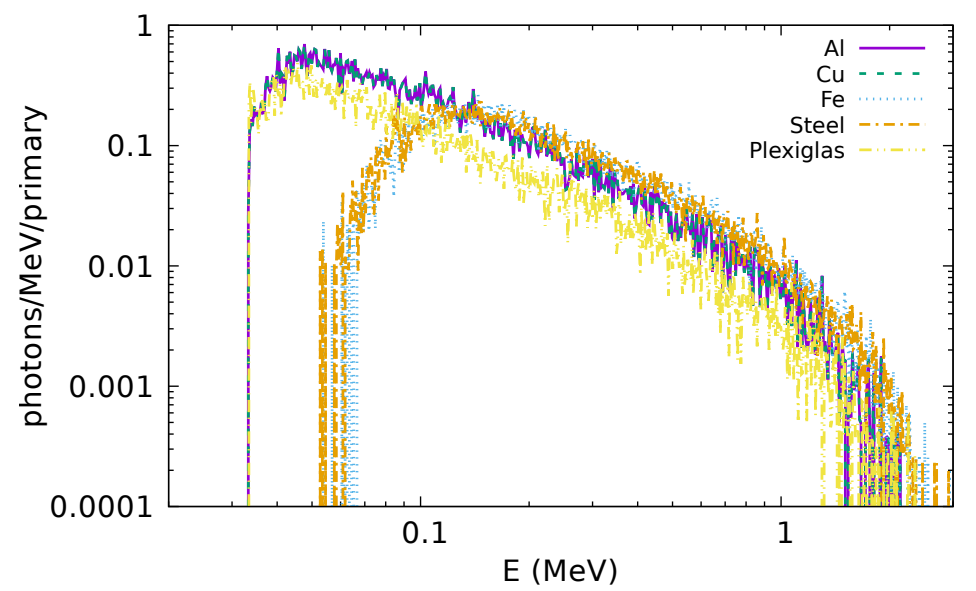

Figure 9: Spectrum of bremsstrahlung radiation produced by a $2 \mathrm{MeV}$ electron beam with $50 \%$ energy spread after interaction with different materials of $5 \mathrm{~mm}$ thickness.

In conclusion, we have investigated theoretically and experimentally the properties of wide-angle electron beams produced by laser-wakefield accelerators in the process leading to the bubble formation. We show that beams with 1-2 MeV energy and $\mathrm{nC}$ level charge are emitted in a forward cone with aperture angle of about $30^{\circ}-50^{\circ}$ from the laser propagation axis. This cone is hollow when plasma electrons are not injected into the bubble and fills in with more energetic electrons when injection occurs and high-energy forward beams are produced. Oblique electrons therefore appear as a high-charge background halo around the high-energy short bunches accelerated inside the bubble. Because of the high charge, wide-angle beams can generate a significant amount of bremsstrahlung radiation and they can cause damage to equipments, which is a particular concern for laser-plasma accelerators based on capillaries. Wide-angle electron beams can also carry a significant fraction of laser energy out of the plasma, limiting the efficiency of the accelerator. 


\section{ACKNOWLEDGMENTS}

We acknowledge support of the U.K. EPSRC (grant no. EP/J018171/1, EP/J500094/1 and EP/N028694/1), the EC's LASERLAB-EUROPE (grant no. 654148), EuCARD-2 (grant no. 312453), EuPRAXIA (grant no. 653782) and the Extreme Light Infrastructure (ELI) European Project. Z.M.S. acknowledges the support of a Leverhulme Trust Grant and the National Basic Research Program of China (2013CBA01504). The authors would like to thank the OSIRIS consortium (UCLA/IST) for the use of OSIRIS. Computational results were obtained using the EPSRC funded ARCHIE-WeSt High Performance Computer (www.archie-west.ac.uk) EPSRC grant no. EP/K000586/1. Data associated with research published in this paper is available at http://dx.doi.org/10.15129/16e6a9b3-a930-48b6-8b1c-72805a7e8850.

\section{REFERENCES}

[1] Mangles, S. et al., "Monoenergetic beams of relativistic electrons from intense laser-plasma interactions," Nature 431, 535-538 (2004).

[2] Geddes, C. et al., "High-quality electron beams from a laser wakefield accelerator using plasma-channel guiding," Nature 431, 538-541 (2004).

[3] Faure, J. et al., "A laser-plasma accelerator producing monoenergetic electron beams," Nature 431, 541-544 (2004).

[4] Leemans, W. P. et al., "Gev electron beams from a centimetre-scale accelerator," Nature Phys. 2, 696-699 (2006).

[5] Esarey, E., Schroeder, C. B., and Leemans, W. P., "Physics of laser-driven plasma-based electron accelerators," Rev. Mod. Phys. 81, 1229-1285 (2009).

[6] Leemans, W. P., Gonsalves, A. J., Mao, H.-S., Nakamura, K., Benedetti, C., Schroeder, C. B., Tóth, C., Daniels, J., Mittelberger, D. E., Bulanov, S. S., Vay, J.-L., Geddes, C. G. R., and Esarey, E., "Multi-gev electron beams from capillary-discharge-guided subpetawatt laser pulses in the self-trapping regime," Phys. Rev. Lett. 113, 245002 (Dec 2014).

[7] Leemans, W. P., Geddes, C. G. R., Faure, J., Tóth, C., van Tilborg, J., Schroeder, C. B., Esarey, E., Fubiani, G., Auerbach, D., Marcelis, B., Carnahan, M. A., Kaindl, R. A., Byrd, J., and Martin, M. C., "Observation of terahertz emission from a laser-plasma accelerated electron bunch crossing a plasma-vacuum boundary," Phys. Rev. Lett. 91, 074802 (Aug 2003).

[8] Schlenvoigt, H. P. et al., "A compact synchrotron radiation source driven by a laser-plasma wakefield accelerator," Nature Phys. 4, 130-133 (2008).

[9] Fuchs, M. et al., "Laser-driven soft-x-ray undulator source," Nature Phys. 5, 826-829 (2009).

[10] Cipiccia, S. et al., "Gamma-rays from harmonically resonant betatron oscillations in a plasma wake," Nat Phys 7, 867-871 (Nov 2011).

[11] Maier, A. R., Meseck, A., Reiche, S., Schroeder, C. B., Seggebrock, T., and Grüner, F., "Demonstration scheme for a laser-plasma-driven free-electron laser," Phys. Rev. X 2, 031019 (Sep 2012).

[12] Corde, S., Ta Phuoc, K., Lambert, G., Fitour, R., Malka, V., Rousse, A., Beck, A., and Lefebvre, E., "Femtosecond x rays from laser-plasma accelerators," Rev. Mod. Phys. 85, 1-48 (Jan 2013).

[13] Jaroszynski, D., Ersfeld, B., Islam, M., Brunetti, E., Shanks, R., Grant, P., Tooley, M., Grant, D., Gil, D., Lepipas, P., McKendrick, G., Cipiccia, S., Wiggins, S., Welsh, G., Vieux, G., Chen, S., Aniculaesei, C., Manahan, G., Anania, M.-P., Noble, A., Yoffe, S., Raj, G., Subiel, A., Yang, X., Sheng, Z., Hidding, B., Issac, R., Cho, M.-H., and Hur, M., "Coherent radiation sources based on laser driven plasma waves," in [Infrared, Millimeter, and Terahertz waves (IRMMW-THz), 2015 40th International Conference on], 1-4 (Aug 2015).

[14] Loulergue, A., Labat, M., Evain, C., Benabderrahmane, C., Malka, V., and Couprie, M. E., "Beam manipulation for compact laser wakefield accelerator based free-electron lasers," New Journal of Physics 17(2), 023028 (2015).

[15] Malka, V., Faure, J., and Gauduel, Y. A., "Ultra-short electron beams based spatio-temporal radiation biology and radiotherapy," Mutation Research/Reviews in Mutation Research 704(1-3), 142-151 (2010). 
[16] Gauduel, Y. A., Glinec, Y., Rousseau, J.-P., Burgy, F., and Malka, V., "High energy radiation femtochemistry of water molecules: early electron-radical pairs processes," Eur. Phys. J. D 60(1), 121-135 (2010).

[17] Subiel, A. et al., "Dosimetry of very high energy electrons (vhee) for radiotherapy applications: using radiochromic film measurements and monte carlo simulations," Physics in Medicine and Biology 59(19), 5811 (2014).

[18] Guillaume, E., Döpp, A., Thaury, C., Lifschitz, A., Goddet, J.-P., Tafzi, A., Sylla, F., Iaquanello, G., Lefrou, T., Rousseau, P., Phuoc, K. T., and Malka, V., "Physics of fully-loaded laser-plasma accelerators," Phys. Rev. ST Accel. Beams 18, 061301 (Jun 2015).

[19] Brenner, C. M., Mirfayzi, S. R., Rusby, D. R., Armstrong, C., Alejo, A., Wilson, L. A., Clarke, R., Ahmed, H., Butler, N. M. H., Haddock, D., Higginson, A., McClymont, A., Murphy, C., Notley, M., Oliver, P., Allott, R., Hernandez-Gomez, C., Kar, S., McKenna, P., and Neely, D., "Laser-driven x-ray and neutron source development for industrial applications of plasma accelerators," Plasma Physics and Controlled Fusion 58(1), 014039 (2016).

[20] McGuffey, C., Thomas, A. G. R., Schumaker, W., Matsuoka, T., Chvykov, V., Dollar, F. J., Kalintchenko, G., Yanovsky, V., Maksimchuk, A., Krushelnick, K., Bychenkov, V. Y., Glazyrin, I. V., and Karpeev, A. V., "Ionization induced trapping in a laser wakefield accelerator," Phys. Rev. Lett. 104, 025004 (Jan 2010).

[21] Pak, A., Marsh, K. A., Martins, S. F., Lu, W., Mori, W. B., and Joshi, C., "Injection and trapping of tunnel-ionized electrons into laser-produced wakes," Phys. Rev. Lett. 104, 025003 (Jan 2010).

[22] Chen, L. M., Yan, W. C., Li, D. Z., Hu, Z. D., Zhang, L., Wang, W. M., Hafz, N., Mao, J. Y., Huang, K., Ma, Y., Zhao, J. R., Ma, J. L., Li, Y. T., Lu, X., Sheng, Z. M., Wei, Z. Y., Gao, J., and Zhang, J., "Bright betatron x-ray radiation from a laser-driven-clustering gas target," Sci. Rep. 3, 1912 (2013).

[23] Kaganovich, D., Gordon, D. F., and Ting, A., "Observation of large-angle quasimonoenergetic electrons from a laser wakefield," Phys. Rev. Lett. 100, 215002 (May 2008).

[24] Zhang, L., Chen, L., Wang, W., Yan, W., Yuan, D., Mao, J., Wang, Z., Liu, C., Shen, Z., Faenov, A., Pikuz, T., Li, D., Li, Y., Dong, Q., Lu, X., Ma, J., Wei, Z., Sheng, Z., and Zhang, J., "Electron acceleration via high contrast laser interacting with submicron clusters," Applied Physics Letters 100(1) (2012).

[25] Yang, X., Brunetti, E., Gil, D. R., Welsh, G. H., Li, F. Y., Cipiccia, S., Ersfeld, B., Grant, D. W., Grant, P. A., Islam, M. R., Tooley, M. P., Vieux, G., Wiggins, S. M., Sheng, Z. M., and Jaroszynski, D. A., "Three electron beams from a laser-plasma wakefield accelerator and the energy apportioning question," Scientific Reports 7, 43910 (2017).

[26] Fonseca, R. et al., "Osiris: A three-dimensional, fully relativistic particle in cell code for modeling plasma based accelerators," in [Computational Science-ICCS 2002, Pt III, Proceedings], 2331, 342-351 (2002).

[27] Lu, W. et al., "Generating multi-gev electron bunches using single stage laser wakefield acceleration in a 3d nonlinear regime," Phys. Rev. ST Accel. Beams 10 (2007).

[28] Jaroszynski, D. et al., "Radiation sources based on laser-plasma interactions," Philos. Trans. R. Soc. A 364, 689-710 (2006).

[29] Devic, S., "Radiochromic film dosimetry: Past, present, and future," Physica Medica 27(3), 122 - 134 (2011).

[30] Ferrari, A., Sala, P. R., Fasso, A., Ranft, J., Européenne, O., La, P., Nucléaire, R., Ferrari, A., Sala, P. R., Fassò, A., and Ranft, J., "Fluka: a multi-particle transport code," in [CERN 2005-10 (2005), INFN/TC 05/11, SLAC-R-773],

[31] Böhlen, T., Cerutti, F., Chin, M., Fassò, A., Ferrari, A., Ortega, P., Mairani, A., Sala, P., Smirnov, G., and Vlachoudis, V., "The FLUKA code: Developments and challenges for high energy and medical applications," Nuclear Data Sheets 120, 211 - 214 (2014). 